\title{
Is there liquid-vapor opalescence at the Mott transition?
}

Scaling theory of the Mott transition and breakdown of Grüneisen scaling near a finitetemperature critical end point

Authors: L. Bartosch, M. de Souza, M. Lang

arXiv:1004.4898

\section{Recommended with a Commentary by Jörg Schmalian, Ames Laboratory and Iowa State University}

Strong electron-electron Coulomb repulsion can localize charge carriers and lead to a Mott insulating state. While charge excitations of the Mott insulator are frozen (gapped), spin degrees of freedom lead to rich magnetic properties at low temperatures. Deep in the Mott insulating state the band-width is small compared to the Coulomb interaction. If one increases the band-width by applying external pressure, $p$, charge fluctuations become more favorable until a first order phase transition to a metallic state takes place. Metals near a Mott transition have attracted much interest as they tend to superconduct (see the organic charge transfer salts and the cuprates). As illustrated in Fig.1, experiment shows that the first order phase transition line terminates at a critical end-point $\left(T_{c}, p_{c}\right)$. Thus, at least for finite $T$, the states on either side of the transition have the same symmetry. Until now the universality class of the end point of the Mott transition is unresolved.

Fig.1 is reminiscent of the liquid-vapor transition, where the critical opalescence near the end-point can be described in terms an Ising order parameter $\varphi$. Close to $p_{c}$ pressure acts like a field $\propto p-p_{c}$ that is conjugate to $\varphi$. The jump $\Delta \varphi$ at the $1^{\text {st }}$-order transition should then vanish as $\Delta \varphi \propto\left(T_{c}-T\right)^{\beta}$, while $\partial \varphi /\left.\partial p\right|_{p_{c}} \propto\left(T-T_{c}\right)^{-\gamma}$ and $\varphi \propto\left|p-p_{c}\right|^{1 / \delta}$, with usual critical exponents $\beta, \gamma$ and $\delta$, respectively. At the liquid-vapor transition, $\varphi$ corresponds to the jump of the fluid density. Similarly, at the Mott transition, $\varphi$ is expected to be proportional to the jump of a local physical observable. Since no symmetry is broken for finite $T$, the jump of $\varphi$ at the $1^{\text {st }}$-order transition is between two finite values. Thus, if $\varphi$ is an order parameter, any analytic function $f(\varphi)$ will equally serve as the order parameter since $\Delta f \propto \Delta \varphi$. A possible, but by no means unique choice is the double occupancy $\left\langle n_{i \uparrow} n_{i \downarrow}\right\rangle$ at lattice site $i$. One further expects that the volume on the metallic side of the transition is different from that on the insulating side:

$$
\Delta \varphi \propto \frac{\Delta V}{V},
$$




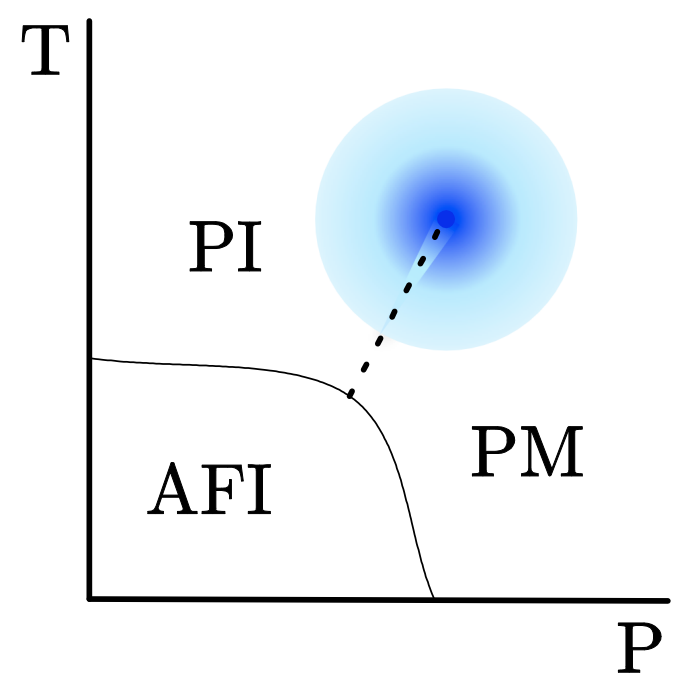

FIG. 1: Schematic phase diagram of Mott transitions as function of pressure and temperature (from Ref.[7])

i.e. $\Delta V / V$ could equally serve as the order parameter.

Experiments and theoretical arguments on Cr-doped $\mathrm{V}_{2} \mathrm{O}_{3}$ support the view that the end-point of the Mott transition is Ising like $\mathrm{e}^{1-3}$. There is however one crucial difference between the Mott transition and the liquid vapor transition: The Mott transition takes place in a rigid crystal with finite shear modulus. Then, elastic forces completely suppress critical fluctuations of an order parameter that obeys Eq.1, and we expect mean field Ising exponents $^{4}$

$$
(\beta, \gamma, \delta)_{\text {mean field }}=\left(\frac{1}{2}, 1,3\right) .
$$

This is essentially what was observed for Cr-doped $\mathrm{V}_{2} \mathrm{O}_{3}$, a three dimensional Mott insulator ${ }^{3}$.

Bartosch, de Souza, and Lang perform an analysis of the thermal expansion $\alpha_{t h}=$ $V^{-1} \partial V / \partial T$ close to the critical end-point of the Mott transition in quasi two-dimensional organic charge transfer salts. Starting from Eq.1, they find very strong evidence for $d=2$ Ising critical behavior, i.e. the exponents are $(\beta, \gamma, \delta)_{d=2 \text {,Ising }}=\left(\frac{1}{8}, \frac{7}{4}, 15\right)$. To this end they compare experimental results of $\alpha_{t h}(T, p)$ with the scaling function that follows from the exact solution of the two dimensional Ising model. As the scaling function depends explicitly on the critical exponents, they conclude from the agreement of theory and experiment that the exponents $2 d$-Ising like. 
This finding is at odds with Eq.2. An explanation of the results by Bartosch et al. could be based on Ref. ${ }^{5}$. Here it was shown that, despite the finite shear rigidity, non-meanfield, $d=2$ Ising criticality results from hexatic melting and disclination unbinding of the underlying crystalline lattice. This scenario can be tested by measuring the structure factor close to the critical point. It would be rooted in the soft lattice and the strong anisotropy of the organic salts.

Finally, the results of this paper seem inconsistent with the data by Kagawa et al. who find yet another set of exponents $(\beta, \gamma, \delta)=(1,1,2)$ in the same class of quasi $2 d$-materials via conductivity and NMR measurements ${ }^{6}$. While measurements of the conductivity (a non-local quantity!) are reconcilable with $d=2$ Ising criticality $^{7}$, the recent measurement of $\delta \simeq 2$ from the jump of the spin lattice relaxation rate near $\left(T_{c}, p_{c}\right)$ is evidence that there are major unresolved issues (see also Ref. ${ }^{8}$ ). Clearly, the critical endpoint of the Mott transition is a beautiful example where strongly correlated electron physics and sophisticated elastic behavior conspire to lead to very interesting classical criticality.

1 D. B. McWhan and J. P. Remeika, Phys. Rev. B 2, 3734 (1970).

2 C. Castellani et al., Phys. Rev. Lett. 43, 1957 (1979); G. Kotliar et al. Phys. Rev. Lett. 84, 5180 (2000).

3 P. Limelette, et al., Science 302, 89 (2003).

4 R. A. Cowley, Phys. Rev. B 13, 4877 (1976).

5 T. Chou and D. Nelson, Phys. Rev. E 53, 2560 (1996).

6 F. Kagawa, K. Miyagawa and K. Kanoda, Nature 436, 534 (2005); Nature Phys. 5, 880 (2009).

7 S. Papanikolaou et al., Phys. Rev. Lett. 100, 026408 (2008).

8 M. Imada, T. Misawa, Y. Yamaji, J. of Phys. Cond. Mat. 22, 164206 (2010) 